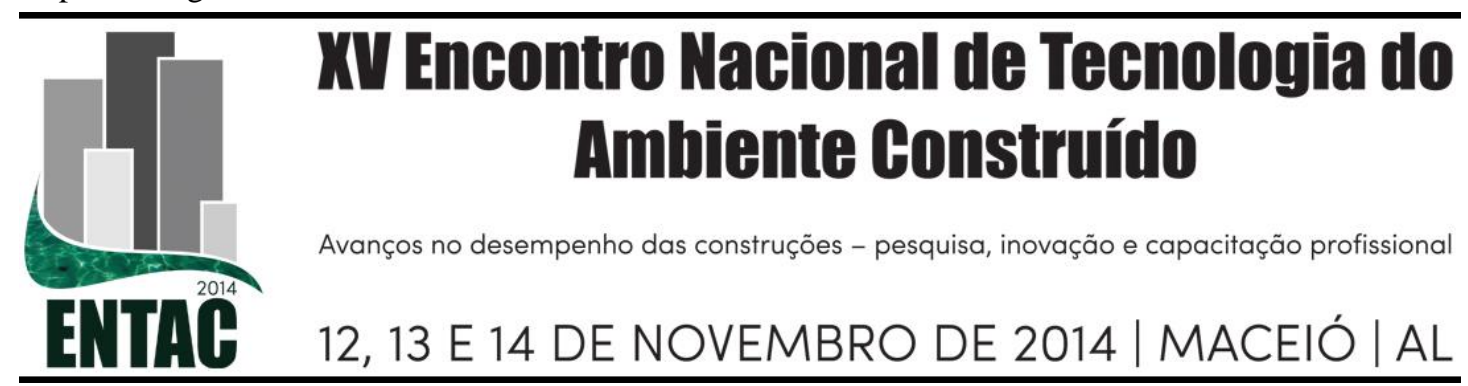

\title{
ANÁLISE DO PROCESSO ORGANIZACIONAL DE PROVISÃO DE HIS: CASO DE LONDRINA-PR
}

TAUBE, Jaqueline(1); HIROTA, Ercília Hitomi(2)

(1) Universidade Estadual de Londrina-UEL, e-mail: jaquetaube@hotmail.com (2)

Universidade Estadual de Londrina-UEL, e-mail: ercilia@uel.br’

\begin{abstract}
RESUMO
Pesquisas indicam que as Habitações de Interesse Social - HIS desenvolvidas no Brasil não tem atendido as reais necessidades dos clientes finais, o que gera a necessidade de adaptações no ambiente construído feitas pelos próprios usuários resultando em habitações com baixa qualidade. Uma solução alternativa seria utilizar a estratégia de customização em massa - CM na produção de casas pré-fabricadas que permite proporcionar aos clientes finais uma gama de opções de produtos, com base nas suas necessidades, com custos e tempo de entrega similar ao alcançado na produção em massa, e assim afetar de forma significativa a maior retenção e satisfação dos clientes atendidos. Este artigo tem como objetivo identificar os pontos críticos do processo de seleção e provisão de habitações a famílias com renda inferior a $\mathrm{R} \$ 1.600,00$ e discutir os pontos propícios à implementação da $\mathrm{CM}$ a partir do mapeamento do fluxo organizacional da Companhia de Habitação da cidade de Londrina-PR, COHAB_LD, companhia responsável pelo cadastro e provisão de HIS na região de Londrina. O artigo apresenta resultados parciais de pesquisa vinculada a dissertação de mestrado da autora principal. A estratégia de pesquisa adotada é o estudo de caso realizado na COHAB_LB. Como resultado, este artigo apresenta os pontos críticos do fluxo organizacional assim como oportunidades para proporcionar a customização em massa para habitação de interesse social.
\end{abstract}

Palavras-chave: Customização em massa, Habitação de Interesse Social, Mapeamento de processo.

\begin{abstract}
Research indicates that Low Income Housing developed in Brazil has not been effective in attending the final customers' real necessities. As a consequence, adaptations in the built environment are made by the dweller, resulting in low quality housing. An alternative solution the use Mass Customization strategy$M C$ to the production of prefab houses. The MC can provide a wide range of product options to the final customers, based on their necessities, with cost and schedule similar to the mass production, therefore, affecting significantly the satisfaction and retention of clients served. The purpose of this article is to identify critical points of housing provision and selection process focused on families with income below $R \$ 1600,00$, as well as discuss propitious points to the implementation of $M C$ in the organizational flow mapping of the Housing Company of Londrina-PR, COHAB_LD. This company is responsible for the records and provision of SIH in Londrina. This article shows the partial results from a research linked to the main author Master Thesis. The research strategy adopted is the case study performed at COHAB_LD. Finally, this article shows the critical points of the organizational flow as well as opportunities in order to provide the mass customization into social interests housing development.
\end{abstract}

Keywords: Mass customization, Social Interest Housing, Process Mapping.

\section{INTRODUÇÃO}

Nas últimas décadas, a comunidade acadêmica tem desenvolvido diversos estudos sobre as habitações de interesse social (HIS) brasileiras, tema este de grande importância econômica e social, já que o país apresenta um amplo déficit habitacional, que afeta 
fortemente a população de menor renda (BRANDÃO, 2002; CIUFFOLINI; SHIMBO, 2013). Muitos destes estudos têm como foco principal a melhoria destas unidades habitacionais, visto que muitos deles revelam que as HIS não têm atendido as reais necessidades dos diversos usuários destes empreendimentos (BONATTO et al., 2011; CIUFFOLINI; SHIMBO, 2013; LIMA et al., 2011).

Os empreendimentos habitacionais de interesse social (EHIS), em sua maioria, são constituídos por unidades habitacionais padrão (BONATTO et al., 2011; BRITO et al., 2011; CIUFFOLINI; SHIMBO, 2013; LIMA et al., 2011) que muitas vezes são replicadas em diversas partes do país, desconsiderando não só as diferentes características climáticas, geográficas e culturais (CIUFFOLINI; SHIMBO, 2013) como também as diferenças de cada família. Também não se observam diferenças entre as unidades dos diferentes programas habitacionais (BONATTO et al., 2011).

As unidades habitacionais padrão, unidades estereotípicas, definidas a partir de um perfil familiar-padrão, não são adequadas (BRANDÃO, 2002), pois tem se observado, nos últimos anos, variação na composição familiar e no tamanho das famílias; mudanças socioeconômicas, inclusão da possibilidade de trabalho em casa e também o desenvolvimento tecnológico de eletrodomésticos, equipamentos para o lar e a inserção do computador no ambiente familiar (BRANDÃO, 2002; LEITE; HEINECK, 2012). Essas constatações evidenciam que as famílias possuem necessidades diferentes e são levadas a fazer modificações e ampliações em suas casas para que as unidades se adequem às suas necessidades. Contudo estas unidades também não são preparadas para tais intervenções, o que acarreta baixa qualidade e alto custo (BRANDÃO, 2011).

A necessidade por mais espaço devido à inadequação do espaço habitacional foi identificada por Bonatto et al. (2011) como o principal motivo para os usuários não permanecerem nos imóveis, enquanto que a possibilidade de ampliações e reformas é vista como um ponto que caracteriza melhoria no estilo de vida dos usuários.

Além destes problemas, os EHIS apresentam, também, baixa qualidade arquitetônica (BRANDÃO, 2011; BRITO et al., 2011; CIUFFOLINI; SHIMBO, 2013; LIMA et al., 2011) razão de forte crítica da produção habitacional brasileira (LIMA et al., 2011). Todos estes problemas acarretam em reclamações e insatisfação dos usuários (LIMA et al., 2011) e, por consequência, ocasionam também baixa retenção do imóvel, o que prejudica as programas habitacionais.

Logo, verifica-se a necessidade de agregar mais valor ao produto. Uma alternativa é utilizar a estratégia de customização em massa - CM na produção de casas préfabricadas. A CM pode ser defina como uma estratégia que permite proporcionar aos clientes finais uma gama de opções de produtos, com base nas suas necessidades, com custos similares ao alcançado na produção em massa (HART, 1995; PINE, 1993) e, assim, afetar de forma significativa a maior retenção e satisfação dos clientes atendidos.

Com base nesta problemática o objetivo deste trabalho consiste em identificar os pontos críticos do processo de seleção e provisão de habitações a famílias com renda até $\mathrm{R} \$ 1.600,00$ e discutir os pontos propícios à implementação da $\mathrm{CM}$ a partir de um estudo de caso do mapeamento do fluxo organizacional da companhia de habitação da cidade de Londrina-PR, COHAB_LD, companhia responsável pelo cadastro e provisão de HIS na região de Londrina. Atualmente, 15.500 famílias com renda até 1,5 salários mínimos, não possuem habitação na cidade de Londrina-PR (COHAB_LD/2013).

Para o desenvolvimento deste trabalho, foi realizada, primeiramente, a pesquisa bibliográfica para uma clara compreensão do conceito de $\mathrm{CM}$ e suas implicações no 
contexto da construção habitacional. A coleta de dados foi realizada por meio de análise de documentos da COHAB_LB, a partir do cadastro da população interessada até o momento de entrega das unidades habitacionais; entrevistas com a responsável pelo Departamento de Assistência Social da companhia e por este processo, e mapeamento do processo de provisão.

\section{CUSTOMIZAÇÃO EM MASSA - CM}

O termo customização em massa foi, primeiramente, discutido por Stanley Davis em 1987, no seu livro Future Perfect onde ele argumenta que os produtos poderiam ser fabricados com as especificações dos clientes, como na economia pré-industrial; e com o custo de um item produzido em massa, como na economia de mercado de massa (DURAY et al., 2000; SILVEIRA et al., 2001).

Pine (1993) define CM como uma estratégia que permite a produção de uma ampla variedade a um baixo custo e complementa ainda que esta é uma nova visão dos negócios, que tem como objetivo identificar e satisfazer os desejos e as necessidades individuais dos clientes sem sacrificar a eficiência, eficácia ou o baixo custo. Pine argumenta ainda, juntamente a outros autores, que esses objetivos da CM são atingidos com o uso de tecnologias de informação e processos flexíveis de trabalho. (GILMORE; PINE, 1997, PINE et al., 1995).

Já para Hart (1995) o conceito tem duas definições distintas. A primeira delas é definida como a capacidade de fornecer o que os clientes querem, quando, onde e como eles querem. Trata-se de um conceito visionário, pois as empresas não conseguem alcançálo, em sua plenitude. A outra é definida como a capacidade de fornecer aos clientes uma variedade de produtos, que podem ser customizados individualmente, a partir da necessidade do cliente, a um custo baixo, assim como no sistema de produção em massa, por meio de estruturas organizacionais e processos flexíveis. Esta é uma definição prática e também mais viável (HART, 1995).

Duray et al. (2000), em um estudo empírico, verificaram que a CM pode ser obtida na prática por meio da modularidade, que permite que os produtos sejam produzidos em larga escala, mantendo os custos de produção mais baixos, e da identificação estratégica do ponto de envolvimento do cliente no processo de desenvolvimento do produto (PDP).

\subsection{Ponto de envolvimento do cliente na customização em massa}

O ponto de envolvimento do cliente é um fator determinante na implementação da $\mathrm{CM}$, já que o atendimento às necessidades específicas dos usuários tende a aumentar significativamente o custo do produto. Duray et al (2000) definem o grau de customização do produto de acordo com o ponto de envolvimento do cliente, no processo de produção: design, fabricação, montagem e uso. Quanto mais cedo, mais customizado será o produto.

Já Mintzberg (1988) apud Duray et al. (2000) que também se apoia neste raciocínio, classifica as formas de customização em três categorias, com os respectivos pontos de envolvimento do cliente:

- Pura: envolvimento do cliente desde o projeto até o uso do produto, altamente customizado; 
- Adaptadas: envolvimento do cliente no ponto fabril, onde os produtos padronizados são alterados para atender as necessidades dos clientes;

- Padronizadas, ou estratégia de customização padronizada: envolvimento do cliente no ponto de montagem e entrega, onde o cliente escolhe as características desejadas a partir de uma lista de opções padrão; apresenta grau baixo de customização, pois a configuração customizada acontece através da organização dos componentes padronizados.

O ponto de envolvimento do cliente, originalmente denominado de Order Penetration Point-OPP, é definido como um ponto na cadeia de produção de um produto, onde o produto é associado à ordem especifica do cliente (OLHAGER, 2003), ou seja, momento em que a ordem do cliente entra na cadeia de valor. Às vezes, também, é chamado de Customer Order Decoupling Point-CODP.

A abordagem do DPD divide o produto em duas partes, a anterior e a posterior a ordem do cliente (FEITZINGER; LEE 1997), essa divisão acontece na determinação do OPP, e a sua posição pode variar de acordo com a estratégia da empresa. $\mathrm{Na}$ etapa anterior devem se concentrar as tarefas da parte padronizada do produto, enquanto que na etapa posterior ao envolvimento do cliente devem se concentrar as tarefas da parte customizada do produto.

A customização a montante aumenta o nível de customização como também o tempo de produção, enquanto que a jusante aumenta o nível de padronização (OLHAGER, 2003). A chave para customizar em massa é adiar a tarefa de diferenciação do produto, para um cliente especifico, até o ultimo ponto possível na rede de abastecimento (FEITZINGER e LEE, 1997). A eficiência do adiamento requer esforço coordenado das equipes de projeto e de produção (ROCHA; KEMMER, 2013).

\section{MÉTODO}

Este artigo apresenta resultados parciais da pesquisa de mestrado em desenvolvimento, da autora principal, cujo objeto de pesquisa é o processo de seleção e provisão de HIS. Para realização desta pesquisa a estratégia utilizada foi o estudo de caso, que segundo Yin (2005) é adequado quando se analisa um fenômeno contemporâneo, em que se busca analisar, de forma detalhada e em profundidade, um fenômeno dentro de seu contexto. Esta estratégia tem a capacidade de lidar com uma ampla variedade de evidências, como, artefatos, entrevistas e observações e na qual o pesquisador tem pouco ou nenhum controle sobre o fenômeno. O estudo foi desenvolvido no Departamento de Assistência Social da Companhia de Habitação de Londrina-PR, COHAB_LD, setor responsável pelo envolvimento com o cliente final, desde o cadastro até provisão de HIS, na cidade de Londrina.

O trabalho foi desenvolvido em quatro etapas. A primeira consistiu em entrevista semiestruturada com a responsável pelo Departamento de Assistência Social da COHAB_LD e coleta de documentos que envolvem os candidatos aos EHIS, desde a etapa de cadastro até o momento de entrega das unidades habitacionais. O objetivo foi identificar como acontece o processo de seleção e provisão da HIS.

$\mathrm{Na}$ segunda etapa, foi realizada análise dos dados coletados na entrevista e nos documentos fornecidos pela companhia; e também elaborado um mapa de fluxo organizacional, atual, do processo de provisão de HIS, com o objetivo de organizar e identificar oportunidades e barreiras para participação dos usuários no processo estudado. 
Já na terceira etapa, foi realizada a validação do mapa do processo de provisão de HIS, dividida em duas fases. A primeira delas constituiu em três rodadas de exposição e discussão do mapa, com a participação de uma arquiteta, mestranda da mesma linha de pesquisa da autora principal deste artigo, que participou da elaboração e aplicação da entrevista. E a segunda foi realizada a partir de exposição e discussão do mapa, seguida de outras duas reuniões com a profissional responsável pelo Departamento de Assistência Social da COHAB_LD: uma para tirar algumas dúvidas que ainda restaram sobre o processo e a outra de validação do mapa. Nos intervalos entre as exposições e discussões o mapa era refeito, visando aprimorar o fluxo, até a validação final. $\mathrm{O}$ objetivo foi elaborar e validar o mapa de fluxo do processo atual.

$\mathrm{Na}$ quarta etapa foi feita a análise do fluxo do processo de provisão da HIS, com a identificação dos pontos críticos do fluxo organizacional e também das oportunidades de customização em massa para HIS.

\section{RESULTADOS E DISCUSSÃO}

A Figura 1 apresenta o fluxo obtido neste estudo. $\mathrm{Na}$ análise dos dados coletados, buscou-se identificar o ponto de envolvimento do cliente, as informações do cliente que entram no processo de provisão, e principalmente, os seus pontos críticos e pontos propícios à implementação da $\mathrm{CM}$.

O processo tem início com a procura dos interessados, clientes/usuários, com idade acima de dezoito anos, junto à $\mathrm{COHAB}$, mais especificamente, ao setor de atendimento ao público da $\mathrm{COHAB}$, para fazer o cadastro na companhia; ou pela mobilização, feita pela própria companhia, das famílias que vivem em áreas de risco, insalubres e/ou irregulares.

Durante o atendimento para o cadastro, é verificada a renda da família e se o interessado possuiu cadastro no Cadastro Nacional de Mutuários - CADMUT da Caixa Econômica, ou seja, se o candidato já fez financiamento de imóvel ou foi beneficiado por programa social de habitação; e no Cadastro Único para programas sociais. A existência de cadastro no CADMUT impede o interessado de ser beneficiado por programa social de habitação. No entanto, ele ainda pode se cadastrar por meio da Ficha de Cadastro de Pessoa Física - FCR da companhia, para financiar $100 \%$ da habitação, caso a companhia tenha empreendimentos para essa população.

Podendo ser beneficiado pelo Programa Minha Casa Minha Vida - PMCMV, o futuro beneficiário preenche a FCR, e informa qual é renda da sua família. Se a renda familiar identificada for de até $\mathrm{R} \$ 1.600,00$, o usuário se enquadra na faixa de habitação prioritariamente atendida pela COHAB-LD e continua no processo de seleção. Caso a renda familiar seja de $\mathrm{R} \$ 1.600,00$ a seis salários mínimos, o usuário continua no processo de seleção, mas em uma outra faixa do PMCMV. Como o foco deste artigo é a faixa de renda até $\mathrm{R} \$ 1.600,00$, as demais faixas são relatadas neste trabalho, somente com intuito de compreender o processo como um todo.

Ainda durante o cadastro, o usuário precisa informar se possui o Cadastro Único, cadastro necessário para participar de programas sociais, e se ele está atualizado. Após finalizar o cadastro, ele recebe as devidas instruções e espera as próximas etapas do processo. Os FCR's são arquivados em ordem numérica.

Antes de abrir um processo de seleção de famílias para um determinado empreendimento, a COHAB_LD faz uma análise de viabilidade, em que se levanta a demanda populacional do local a ser implantado, assim como os equipamentos 
necessários para atender esta população; após análise é emitido um relatório, que permite abrir o processo de seleção, caso haja demanda e os demais quesitos necessários.

Após abertura do processo, são consideradas candidatas apenas as famílias que atendem ao requisito de territorialidade, ou seja, residem no raio de $2,5 \mathrm{Km}$ do local em que será implantado o EHIS. Este processo recomeça a cada empreendimento lançado e os que não atendem a este requisito continuam esperando o próximo ciclo, e assim sucessivamente. Então, são solicitados os documentos comprobatórios de toda a família: pessoais, de renda, composição familiar, endereço e Cadastro Internacional de Doenças - CID, se houver. Aqueles que apresentarem pendências devem sana-las e os que não se enquadrarem nesta faixa do programa devem retornar ao início do processo em outra faixa de atendimento ou sair do processo. Ou seja, novamente são verificados os requisitos das famílias.

$\mathrm{Na}$ próxima etapa, é verificado o atendimento aos critérios do PMCMV, sendo três critérios nacionais (famílias residentes em áreas de risco ou insalubres, com mulheres responsáveis pela unidade familiar, com idosos ou pessoas com deficiência) e três locais (territorialidade, famílias com residência fixa no Município de Londrina pelos últimos cinco anos e em situação de vulnerabilidade social). Nesta etapa são selecionados a continuar neste processo os que atendem a todos os critérios, ou os que mais atenderem conforme demanda e tamanho do empreendimento.

Para os que continuam, neste ciclo do processo, a Assistência Social da COHAB agenda e faz uma visita às famílias para verificar a condição de moradia e os indicadores de vulnerabilidade, de acordo com os critérios estabelecidos pelo IPARDES ${ }^{1}$, em 2012, e os critérios do PMCMV. Após visitas é emitido um parecer social que identifica quais são as famílias prioritárias no processo, mediante a triagem e hierarquização das famílias. De acordo com a ordem hierárquica obtida, a COHAB seleciona as famílias com base no número de unidades a serem oferecidas. O número selecionado é $30 \%$ maior do que o de unidades para que ao final do processo não faltem famílias para as unidades entregues, já que as famílias podem, ainda, apresentar pendências ou, mesmo, não se enquadre em todos os requisitos do programa, verificados nas etapas seguintes. $\mathrm{O}$ público que não é prioridade aguarda os próximos empreendimentos.

Os selecionados são indicados para a Caixa Econômica Federal - CEF fazer análise do perfil das famílias, considerando: o CADMUT, novamente, o Cadastro Nacional de Inadimplência - CADIN e a renda familiar. Em uma etapa paralela a esta o empreendimento em desenvolvimento normalmente já se encontra com $60 \%$ da obra concluída.

Após esta análise do perfil das famílias é encaminhado para a COHAB a lista com os perfis que foram aprovados pela CEF e então a companhia de habitação envia os documentos dos aprovados para nova análise da CEF. Após esta nova análise a CEF encaminha nova lista para a COHAB com o nome dos habilitados para receber as unidades. Aqueles que apresentarem pendências, ou que não se enquadrarem nesta faixa do programa devem retornar ao início do processo em outra faixa de atendimento ou sair do processo.

\footnotetext{
${ }^{1}$ Instituto Paranaense de Desenvolvimento Econômico e Social
} 
Figura 1 - Mapa do fluxo organizacional do processo de provisão de HIS em Londrina-PR

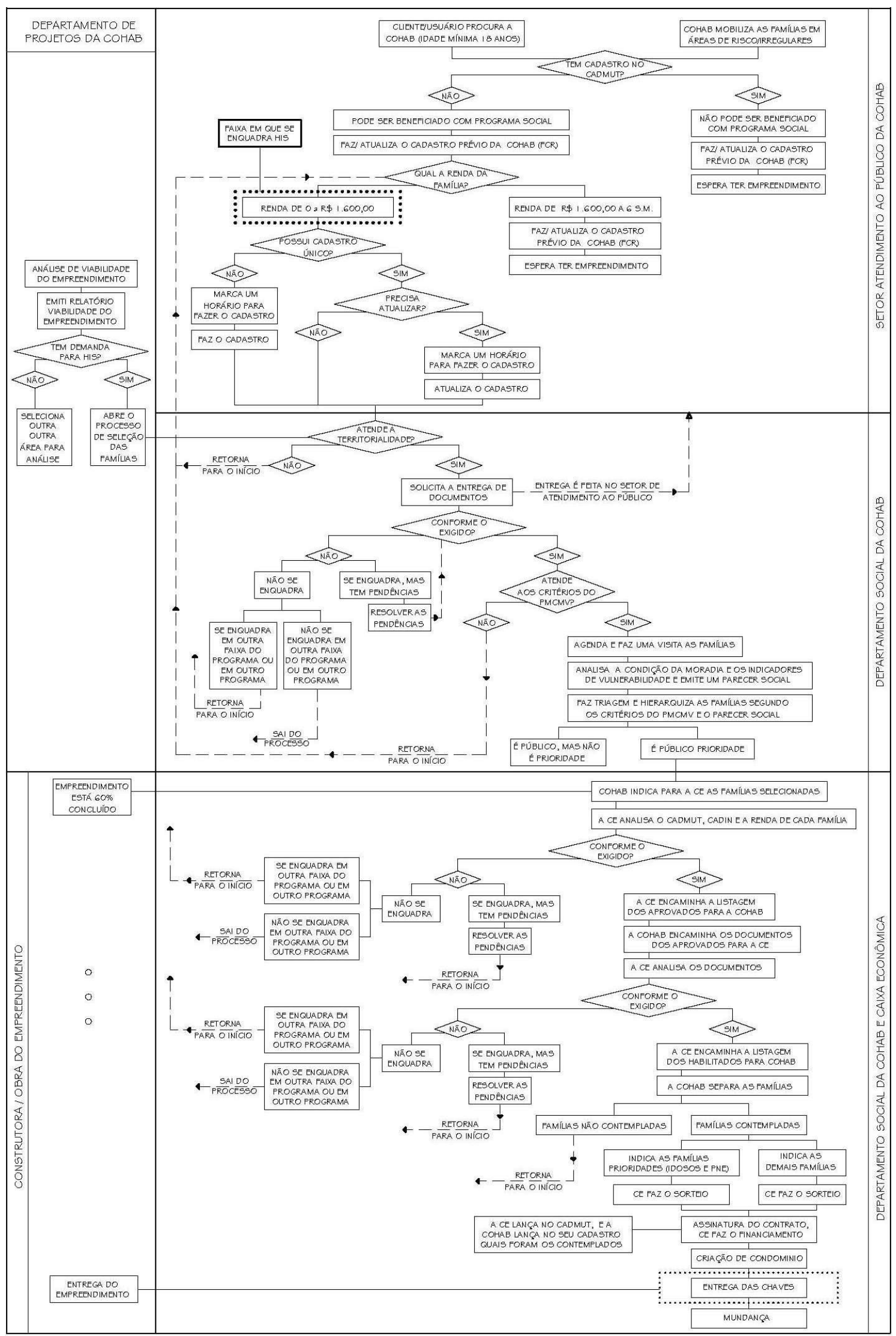


Somente após esta triagem as famílias contempladas são identificadas, mediante a compatibilização entre seleção dos habilitados pela $\mathrm{CEF}$, o número de unidades entregues e a hierarquia estabelecida anteriormente. As famílias não contempladas retornam para o início do processo, enquanto que as contempladas são divididas em 2 grupos: as famílias com pessoas idosas e/ou com necessidades especiais, são consideradas prioritárias para que recebam as casas que possuam melhor acesso, entre a rua e a unidade habitacional, embora todas as unidades sejam projetadas para atendelas; e as demais famílias são contempladas por sorteio das unidades restantes, realizado pela CEF.

O processo é finalizado com a assinatura dos contratos pelas famílias e o lançamento dos dados das mesmas no sistema pela $\mathrm{CEF}$ e $\mathrm{COHAB}$, com consequente entrega das chaves.

A análise das informações coletadas e o mapeamento do processo de seleção e provisão de HIS permitiram identificar, até o momento, que as informações coletadas sobre os usuários são superficiais e que há oportunidades de melhoria no processo.

Com exceção das informações coletadas na FCR sobre as preferências dos clientes quanto ao tipo de imóvel, quantidade de quartos e região de interesse, as demais informações coletadas, se limitam aos dados pessoais, composição, condições de saúde e renda familiar, e as características do domicilio atual. Observa-se, então, que durante o processo não são coletadas informações como o número e as dimensões dos cômodos; área da edificação e os espaços necessários para atender as necessidades de cada família, relacionados à adequação do espaço ambiental, apontado por Bonatto et al. (2011) como o principal motivo para retenção das unidades. Estas informações se referem também as principais modificações levantadas por Brandão (2011).

O mapeamento do processo indica também que a definição das famílias comtempladas só se efetua nas etapas finais, coincidindo com a fase final de execução do empreendimento, o que inviabiliza a consideração de necessidades específicas da família no processo de desenvolvimento do produto. Além disso, a designação das casas é feita por meio de sorteio, pela CEF.

Para aumentar a retenção das famílias nas unidades habitacionais, considera-se importante aumentar a eficácia no atendimento às necessidades dessas famílias, o que implica em abordar, de forma simultânea e convergente os dois processos: de desenvolvimento do produto, incluindo a produção das habitações e de seleção das famílias.

No que se refere ao desenvolvimento do produto, um dos aspectos a ser considerado é a tecnologia construtiva adotada: a tecnologia tradicional para a produção habitacional em larga escala demanda longo período de produção e implica na gestão de uma grande variedade de recursos. Essas duas características impõem sérias restrições à designação de unidades habitacionais específicas a famílias devidamente identificadas.

Entretanto com a adoção de tecnologia pré-fabricada, o OPP do cliente, ou a definição das famílias poderia ser próximo à fase final das unidades, no qual os módulos das unidades seriam definidos pelos clientes, segundo as suas necessidades, em uma fase anterior a de montagem das moradias.

Com relação à seleção das famílias, observa-se o quão segmentado é o processo e que o mesmo poderia ser sintetizado, evitando retrabalhos. A etapa de entrega de documentos feita pelos usuários poderia ser feita junto ao cadastro do interessado, que acarretaria em encurtamento do processo entre as etapas de identificação da territorialidade e a 
primeira lista dos participantes da seleção, público prioridade; assim como o envio do nome das famílias selecionadas poderia ser feita junto com o envio dos documentos, das respectivas famílias, pela $\mathrm{COHAB}$ para as análises da $\mathrm{CEF}$, que reduziria as etapas entre a indicação das famílias feitas pela $\mathrm{COHAB}$ à $\mathrm{CEF}$ e a lista das famílias habilitadas, encaminhada pela $\mathrm{CEF}$ à $\mathrm{COHAB}$. A redução nas etapas do processo de seleção permitiria a antecipação do OPP no PDP. O processo poderia, também, ser adaptado ao OPP do cliente se as ferramentas de coleta de dados fossem adaptadas para, também, capturar os requisitos destes clientes e de como estas seriam inseridas no PDP.

\section{CONSIDERAÇÕES FINAIS}

A CM pode ser uma maneira de agregar mais valor ao produto para o cliente final e desta forma obter maior retenção das unidades. A interação entre os dados coletados do cliente e o ponto de envolvimento com o processo são cruciais para implementar a CM, pois estes proporcionam o conhecimento das necessidades de cada cliente e o momento em que elas são inseridas no PDP, desde que o processo esteja preparado para isso.

Os resultados obtidos até o momento na pesquisa relatada neste artigo mostram que, mesmo dentro das limitações encontradas, é possível implementar melhorias nos processos de seleção de famílias e de desenvolvimento do produto de forma a buscar a CM. Mas para que a implementação desta abordagem seja viável em EHIS é necessário que os produtos ou seus componentes sejam modulares e desta forma possam ser produzidos em larga escala, como na produção em massa, que permite a redução dos custos do produto.

$\mathrm{Na}$ continuidade desta pesquisa, mais uma etapa já foi concluída, na qual foram levantados os requisitos dos clientes por meio de uma Avaliação Pós-Ocupação - APO, realizada em um empreendimento destinado a população com a mesma faixa de renda da população analisada no estudo de caso aqui apresentado. Posteriormente, será realizada uma oficina com experts com o objetivo de delinear o modelo do processo de provisão de HIS e delimitar o escopo de CM para este tipo de habitação, baseado na literatura de $\mathrm{CM}$, nos requisitos dos clientes levantados e no mapeamento do processo apresentado; em seguida, será realizada uma análise cronológica do mapa do processo de provisão de HIS com intuito de sincroniza-lo com o processo de projeto e análise dos OPPs; e então propor um modelo de processo de provisão de HIS adaptado a CM de casas pré-fabricadas. E, por fim, a validação do modelo proposto por profissionais envolvidos no processo de provisão de HIS em Londrina.

\section{AGRADECIMENTOS}

Agradecemos a CAPES, que através de seu Programa de concessão de Bolsas e financiamento à pesquisa tem possibilitado o desenvolvimento de nossos trabalhos; e a COHAB_LB pelas informações fornecidas.

\section{REFERÊNCIAS}

BONATTO, F. S.; MIRON, L. I. G. FORMOSO, C. T. Avaliação de empreendimentos habitacionais de interesse social com base na hierarquia de valor percebido pelo usuário. Revista Ambiente Construído, Porto Alegre, v. 11, n. 1, p. 67-83, jan./mar. 2011.

BRANDÃO, D. Q. Disposições técnicas e diretrizes para projeto de habitações sociais evolutivas. Revista Ambiente Construído, Porto Alegre, v. 11, n. 2, p. 73-96, abr./jun. 2011. 
BRANDÃO, D. Q. Personalização do produto habitacional: novas demandas e resposta das tecnologias de construção. In: ENCONTRO NACIONAL DE ENGENHARIA DE PRODUÇÃO, 22., 2002, Curitiba. Anais... Curitiba: ABEPRO, 2002. p. 1-8.

BRITO, J. N. de S.; FORMOSO, C. T.; ECHEVESTE, M. E. Análise de dados de reclamações em empreendimentos habitacionais de interesse social: estudo no Programa de Arrendamento Residencial. Revista Ambiente Construído, Porto Alegre, v. 11, n. 4, p. 151-166, out./dez. 2011.

COHAB/LD. PMH - Plano Municipal de Habitação: Gestão 2013-2016. Londrina, Paraná, 2013. Disponível em: <http://www1.londrina.pr.gov.br/dados/images/stories/Storage/cohab/ regularizacao_fund $>$. Acesso em: 07 mai. 2014.

CIUFFOLINI, M. A.; SHIMBO, L. Z. Políticas urbanas e habitacionais e seus efeitos sociais. Um estudo do Programa "Minha Casa, Minha Vida" no Brasil e na Argentina. In: BONELLI, M. da G.; LANDA, M. D. V. de (Org.). Sociologia e mudança social no Brasil e na argentina. São Carlos: Compacta Gráfica e Editora, 2013. p. 241-270.

DURAY, R.; WARD, P. T.; MILLIGAN, G. W.; BERRY, W. L. Approaches to mass customizations and empirical validation. Journal of Operations Management, v.18, p.605625,2000 .

FEITZINGER, E.; LEE, H. L. Mass customization at Hewlett-Packard: The Power of Postponement. Harvard Business Review. v.75, n.1, p. 116-121, 1997.

GILMORE, J. H.; PINE, B. J. The four faces of mass customization. Harvard Business Review, v.75, n.1, p.91-97, 1997.

HART, C. W. L. Mass customization: conceptual underpinnings, opportunities and limits. International Journal of Services Industry Management, v.6, n.2, p.36-45, 1995.

LEITE, K. P.; HEINECK, L. F. M. Flexibilidade arquitetônica e o gerenciamento de empreendimentos de habitação de interesse social - HIS. In: ENCONTRO NACIONAL DE TECNOLOGIA DO AMBIENTE CONSTRUÍDO, 14., 2012, Juiz de Fora. Anais... Juiz de Fora: ENTAC, 2012. p. 2827-2836.

LIMA, L. P.; FORMOSO, C. T.; ECHEVESTE, M. E. S. Proposta de um protocolo para o processamento de requisitos do cliente em empreendimentos habitacionais de interesse social. Revista Ambiente Construído, Porto Alegre, v. 11, n. 2, p. 21-37, abr./jun. 2011.

OLHAGER, J. Strategic positioning of the order penetration point. International Journal Production Economics. v. 85, n.3, p. 319-329, 2003.

PINE, B. J. Mass Customizing Products and Services. Strategy \& Leadership, v. 21, n.4, p. 6$55,1993$.

PINE, B. J.; PEPPER, D.; ROGERS, M. Do you want to keep your customers forever?.

Harvard Business Review, p. 103-114, 1995.

ROCHA, C. G. da; KEMMER, S. L. Method to Implement Delayed Product Differentiation in Construction of High-Rise Apartment Building Projects. Journal of Construction Engineering and Management, v.139, n.10, 8p., out.2013.

SILVEIRA, G. da; BORENSTEIN, D.; FOGLIATTO, F. S. Mass customization: Literature review and research directions. International Journal of Production Economics, v.72, n.1, p.1-13, 2001.

YIN, R. K. Estudo de Caso: Planejamento e métodos. 3 ed. Porto Alegre: Bookman, 2005. 